\title{
MWC 560: An SS 433 type object with a white dwarf
}

\author{
T. Iijima ${ }^{\star}$ \\ Astronomical Observatory of Padova, Asiago Section, Osservatorio Astrofisico, 36012 Asiago (Vi), Italy \\ Received 19 February 2002 / Accepted 14 May 2002

\begin{abstract}
Spectral variations of the peculiar symbiotic object MWC 560 (V694 Mon) have been monitored for 10 years since 1991. The radial velocity of the blue edge of the broad absorption components of $\mathrm{H} \mathrm{I}$ lines is measured to evaluate the outflow velocity of the jet. The variation of the outflow velocity suggests that there are active and stable stages in this system. The outflow velocity in the stable stage seems to vary according to the long-term light variation with a period of 1930 days. This variation of the outflow velocity could be explained with a model of gas outflow from an accretion disk precessing with a period of 1930 days. If the observed amplitude of the light variation, $\Delta m_{\mathrm{pg}} \cong 0.72 \mathrm{mag}$, was purely due to the precession, the maximum inclination of the disk to the line of sight should be about 59 degrees. Since the outflow is observed always as absorption, the open angle of the outflow should be larger than 59 degrees. However, the amplitude of the light variation due to the precession of the disk seems to be still rather uncertain. In the active stage the outflow velocity was much higher (in the negative sense) than in the stable stage, and rapid variations of the outflow velocity were observed. During the outburst in 1990, this object was in the active stage, while no significant brightening was observed in the active stages in 1993 and 1999 at least in the $V$ band. The durations of the active stages were several months.
\end{abstract}

Key words. stars: individual: MWC 560 (V694 Mon) - stars: binaries: symbiotic - ISM: jets and outflows

\section{Introduction}

MWC 560 (V694 Mon) is a peculiar symbiotic-like variable star. Its spectrum shows prominent emission lines of $\mathrm{H} \mathrm{I}, \mathrm{He} \mathrm{I}$, Fe II, Ti II, [O I], etc. superimposed on the absorption features of an M type giant (e.g. Tomov et al. 1992; Chentsov et al. 1997; Tomov \& Kolev 1997; Schmid et al. 2001). The uniqueness of this object is due to the highly blue-shifted broad absorption components of H I and some other prominent lines, as reported in previous works (e.g. Michalitsianos et al. 1991; Tomov et al. 1992; Chentsov et al. 1997; Iijima 2000; Schmid et al. 2001).

This object underwent a weak outburst in early 1990 and reached a maximum luminosity of 9.2 mag in $V$ band, then slowly faded in 1991-1992 to the quiescent stage of $V \cong 10.5$ (Tomov et al. 1992; Tomov et al. 1996; Mikolajewski et al. 1997; Iijima 2000). Large and rapid variations of the ultraviolet and optical spectra were observed during the outburst (Michalitsianos et al. 1991; Buckley 1992; Tomov et al. 1992). The maximum blue-shift of the absorption component of $\mathrm{H} \beta$, $-6500 \mathrm{~km} \mathrm{~s}^{-1}$, was observed in March 1990 during the outburst (Szkody \& Mateo 1990).

It has been suggested that this object may be a binary system consisting of an $\mathrm{M}$ type giant and a white dwarf with an accretion disk (Tomov et al. 1992; Panferov et al. 1997; Schmid et al. 2001). Tomov and Kolev (1997) suggested that the binary system may be nearly face-on, because no significant variation

\footnotetext{
^ e-mail: iijima@astras.pd.astro.it
}

of the radial velocities of the absorption lines of the $M$ type giant nor of the emission lines of singly ionized metal was detected. Also, in a recent work by Schmid et al. (2001) no detectable variation of the radial velocities of these elements was noticed. Tomov et al. (1992) classified the M type giant as M4.5 III, while Schmid et al. (2001) proposed a slightly later type, M 5.5 III. It has been suggested that the broad absorption components may be formed in a high velocity jet from an accretion disk around the white dwarf (Tomov et al. 1992; Shore et al. 1994; Panferov et al. 1997; Schmid et al. 2001). However, the behaviour of the broad absorption components is not yet well known. In this work, the results of the monitoring of the spectral variations of this object carried out since 1991 are reported. A model for the long-term variation of the radial velocities of the broad absorption components is presented.

\section{Observation}

The observations were carried out with an Echelle spectrograph mounted on the $182 \mathrm{~cm}$ telescope of the Mount Ekar station of the Astronomical Observatory of Padova. The data reductions were made using NOAO IRAF package at Asiago Observatory. The sensitivity of the spectrograph was corrected using the spectra of spectrophotometric standard stars Hiltner 600 and HD 60778 taken on the same nights. The spectral range of most spectra is from $4300 \AA$ to $6900 \AA$ and the resolution is $\Delta \lambda / \lambda \sim 8000$. The spectrum on February 3, 1991 covers only the $\mathrm{H} \alpha$ region, while those on December 23, 1991 and March 9, 1993 cover only the $\mathrm{H} \beta$ region. A $\log$ of the observations is 


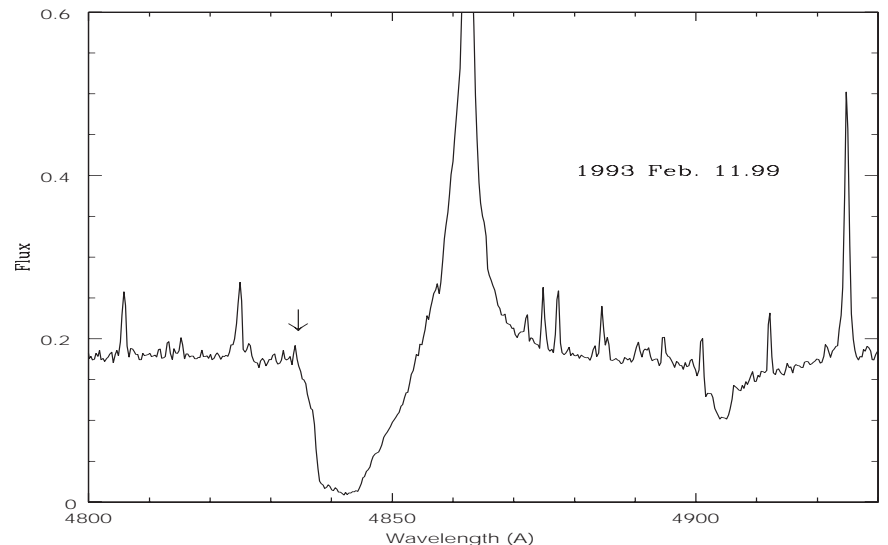

Fig. 1. $\mathrm{H} \beta$ on Feb. 11, 1993. The profile of the absorption component is type A. The arrow indicates its blue edge.

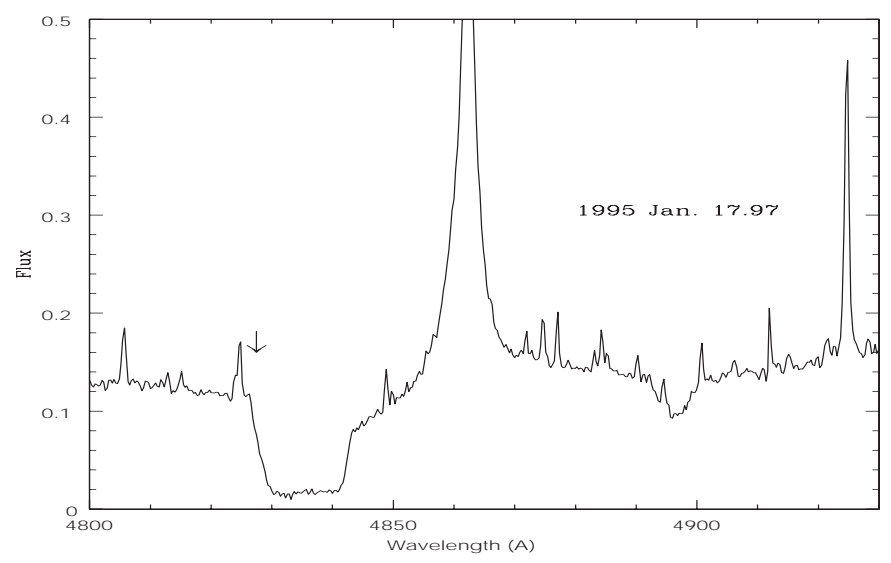

Fig. 2. $\mathrm{H} \beta$ on Jan. 17, 1995. The profile of the absorption component is type $\mathrm{B}$. The arrow indicates its blue edge.

given in Table 1, where UT and JD are universal time and Julian Day -2400000 at mid-exposure. The column "stage" shows whether the object was in the active (act) or stable (stb) stage (see, Sect. 3.2), and the column "profile" presents the type of profile of the broad absorption components of H I lines (see, Sect. 3.1).

The radial velocity of the blue edge, which is indicated by an arrow in Figs. 1-5, of the broad absorption components of $\mathrm{H} \alpha$ and $\mathrm{H} \beta$ were measured to evaluate the outflow velocity $\left(v_{\max }\right)$ of the jet. The radial velocity at the middle of the half depth of the broad absorption components ( $\left.v_{\text {cent }}\right)$ and the full width at half depth $(f w h d)$ were also measured. The observational error in $v_{\max }$ is about $\pm 50 \mathrm{~km} \mathrm{~s}^{-1}$ and those in $v_{\text {cent }}$ and $f w h d$ are about $\pm 20 \mathrm{~km} \mathrm{~s}^{-1}$. The observed results are summarized in Table 1.

\section{Broad absorption components of $\mathrm{H}$ I lines}

\subsection{Profile}

The profiles of the broad absorption components of $\mathrm{H}$ I lines are classified into four types (A, B, C, D). The first, type A, shows no flat continuum between the absorption and emission components, and the blue side is steeper than the red side (Fig. 1). The second, type B, also shows no flat continuum between the

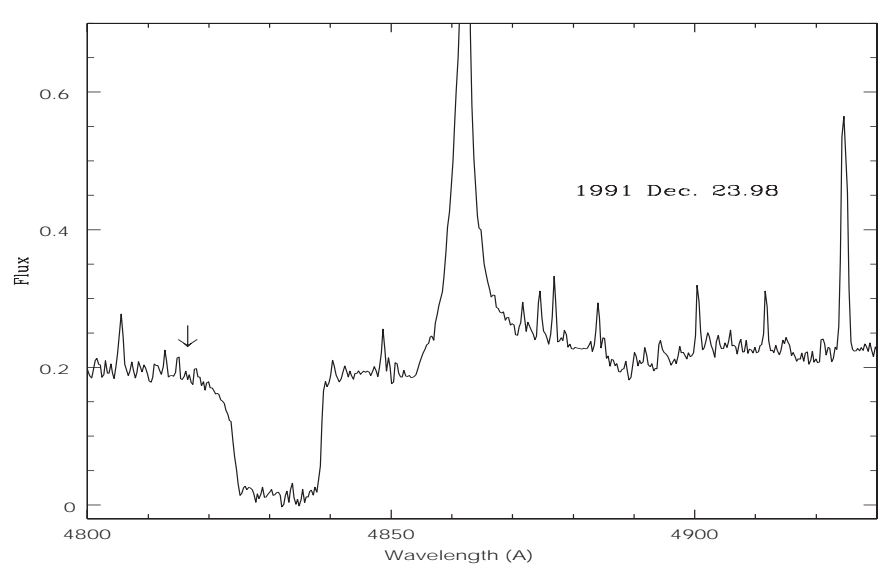

Fig. 3. H $\beta$ on Dec. 23, 1991. The profile of the absorption component is type $\mathrm{C}$. The arrow indicates its blue edge.

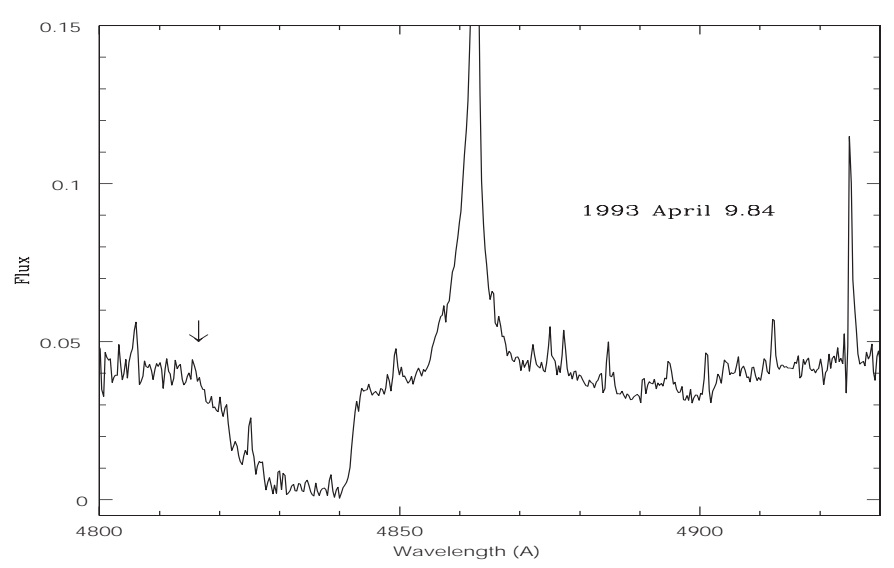

Fig. 4. $\mathrm{H} \beta$ on April 9, 1993. The profile of the absorption component is type $\mathrm{D}$. The arrow indicates its blue edge.

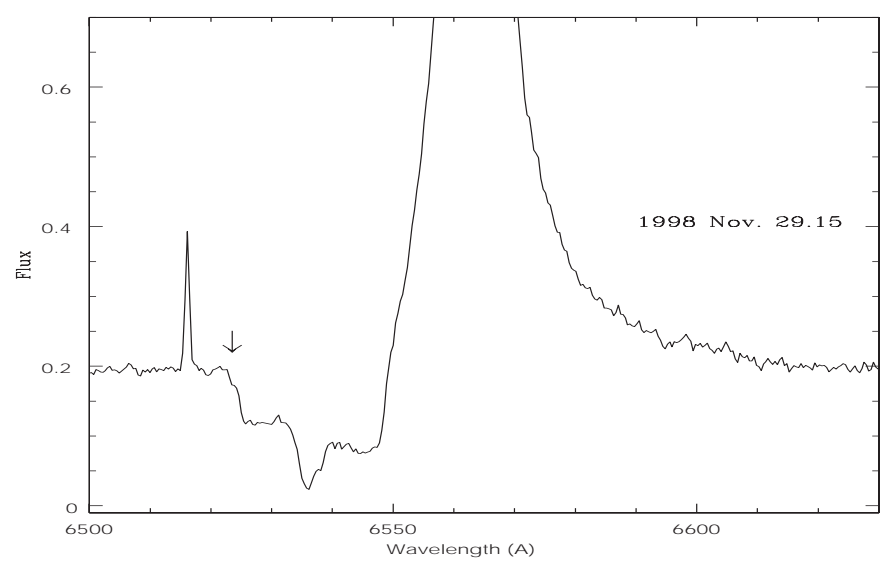

Fig. 5. H $\alpha$ on Nov. 29, 1998. The profile of the absorption component is type $\mathrm{A}+\mathrm{nr}$. The arrow indicates its blue edge.

absorption and emission components, but a part of the red side is as steep as the blue side i.e. there is a clear bending in the slope of the red side. An example is shown in Fig. 2. The third type, $\mathrm{C}$, shows a flat continuum between the absorption and emission components, and both blue and red sides are steep. An example is shown in Fig. 3. The last type, D, is shown in Fig. 4, where a flat continuum is seen. The red side is steeper than the blue side. The types of profile are given in the column 
Table 1. Log of observations and radial velocities in $\mathrm{km} \mathrm{s}^{-1}$ of the broad absorption components of H I lines.

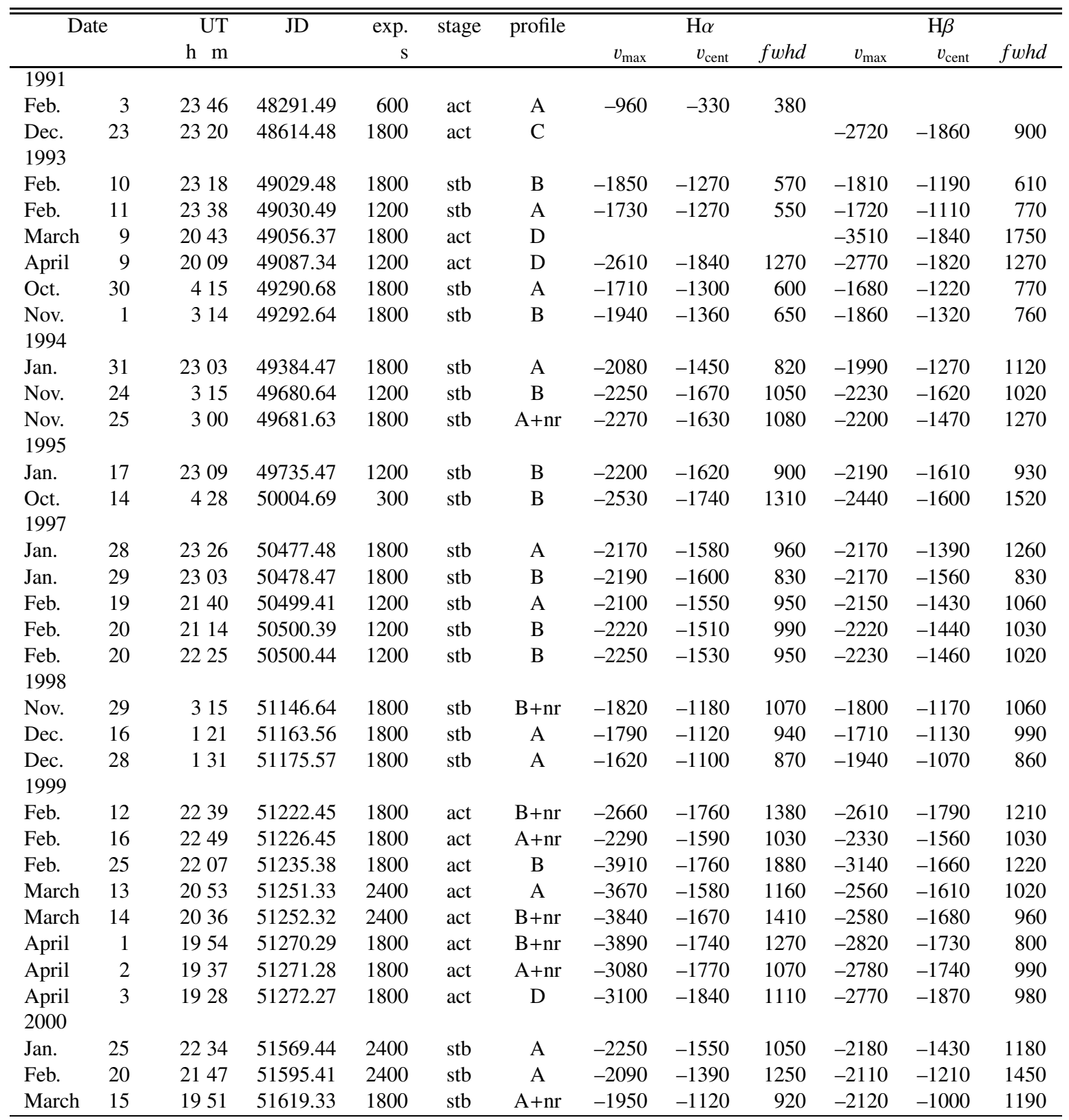

JD: Julian day -2400000 .

"profile" of Table 1. In some spectra, rather narrow absorption components superimposed on the broad absorption components. A term "nr" is added to the type of profile in such cases and an example is shown in Fig. 5. A short report on the narrow absorption components of $\mathrm{H} \mathrm{I}$ lines of this object is given by Iijima (2000), and a detailed analysis will be given in a forthcoming paper.

\subsection{Outflow velocity}

During the outburst in 1990, large blue-shifts of the broad absorption components were observed, and the blue-shifts varied with a time scale of less than one day (Michalitsianos et al. 1991; Buckley 1992; Tomov et al. 1992). Similar high velocities and their rapid variations were observed in 1992 , 1993, and 1999 during our monitoring. Hereafter, we will call these phases the "active stage", whereas the remaining phases will be called the "stable stage". When there was only one observation in a season, it is impossible to know whether there was a rapid variation of the outflow velocity. We used the following criteria for such cases. If the profile of the absorption component was type $\mathrm{A}$ or $\mathrm{B}$, and the outflow velocity agreed well with that expected in the stable stage (see the latter half of this sub-section.), we classified the data into the stable stage. On the other hand, if a profile of type C or D was seen, or if the outflow velocity was largely different (roughly more than $300 \mathrm{~km} \mathrm{~s}^{-1}$ ) from that of the stable stage, we classified the data into the active stage. The column "stage" of Table 1 shows 


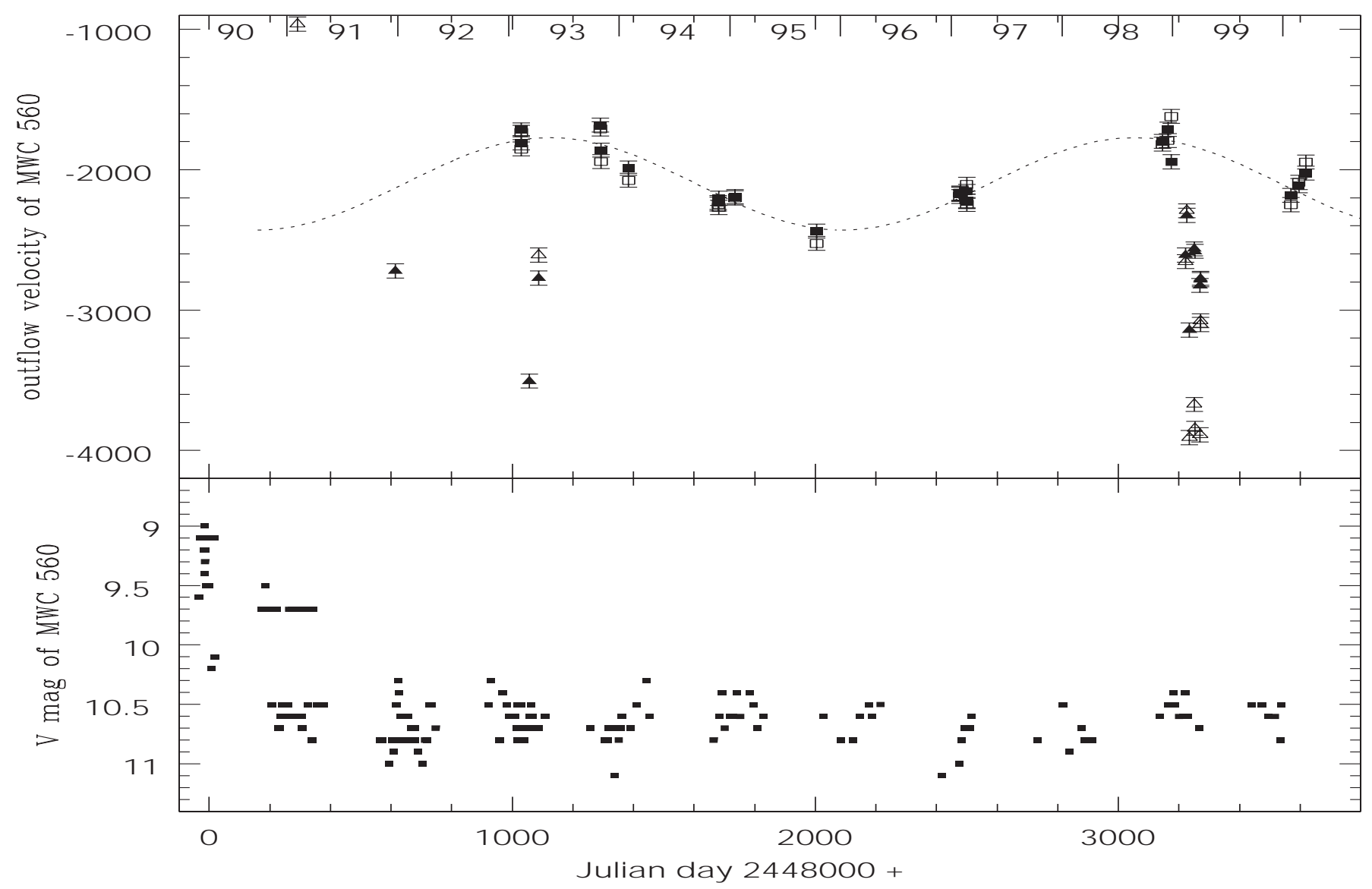

Fig. 6. Outflow velocities in $\mathrm{km} \mathrm{s}^{-1}$ measured on $\mathrm{H} \alpha$ and $\mathrm{H} \beta$. The open square and triangle show the velocities of $\mathrm{H} \alpha$ in the stable and active stages respectively, while the solid symbols are those of $\mathrm{H} \beta$. The dotted line shows the best fit curve for the outflow velocities in the stable stage. The lower panel shows $V$ mag collected from VS-Net.

whether the object was in the active stage (act) or in the stable stage (stb). Figure 6 shows the outflow velocities $\left(v_{\max }\right)$ measured on $\mathrm{H} \alpha$ and $\mathrm{H} \beta$, and the $V$ mag of this object collected from VS-Net ${ }^{1}$. The outflow velocities in the stable stage are shown by open $(\mathrm{H} \alpha)$ and solid $(\mathrm{H} \beta)$ squares, while the triangles are those in the active stage.

The outflow velocity in the stable stage seems to have oscillated slowly in the range between $-1800 \mathrm{~km} \mathrm{~s}^{-1}$ and $-2400 \mathrm{~km} \mathrm{~s}^{-1}$ (Fig. 6). It is interesting that the variation of the outflow velocity in the stable stage may correlate with the long-term light variation with a period of 1930 days found by Doroshenko et al. (1993):

$\mathrm{JD}_{\min }=2437455+1930 \times E$.

Since our data cover only one and half circles of the period of 1930 days, a new periodicity search in the variation of $v_{\max }$ was not made, but the fitting was made assuming the period of 1930 days. The best fit curve for the outflow velocity in the stable stage is given as:

$$
\begin{gathered}
v_{\max }=-2100+330 \cdot \cos \left(2 \pi\left(t-t_{0}\right) / P\right) \mathrm{km} \mathrm{s}^{-1}, \\
\pm 5 \quad \pm 10
\end{gathered}
$$

where $P$ is the period of 1930 days, $t$ is the epoch of observation and $t_{0}$ is the epoch of the lowest (in negative sense)

\footnotetext{
${ }^{1}$ http://www.kusastro.kyoto-u.ac.jp/vsnet/index.html
}

outflow velocity. We found $t_{0}=2447195$ which corresponds to phase 0.047 of the ephemeris of Doroshenko et al. (1993), i.e. the epoch of the lowest outflow velocity nearly coincides with that of light minimum. The best fit curve is shown by a dotted line in Fig. 6.

\section{Gas outflow from a precessing disk}

Tomov \& Kolev (1997) suggested that this object may be a nearly face-on binary system, because no detectable variation of the radial velocities was observed for the absorption lines of the M type giant, nor for the emission lines of singly ionized metals. If this is the case, the periodical light variation reported by Doroshenko et al. (1993) may not have been due to an eclipsing phenomenon, or to the rotation of the $\mathrm{M}$ type giant illuminated by the hot component.

Doroshenko et al. (1993) suggested some possible causes of the periodical light variation, such as orbital motion, accretion disk precession, pulsation of the $\mathrm{M}$ giant etc., and then rather supported a model of a binary system with an elliptical orbit, where the mass accretion rate varies according to the orbital motion. If the mass accretion rate varies with the orbital motion, the luminosity of the accretion disk and the outflow velocity could vary with the same period. It seems, however, to be 
difficult to explain the mass outflow phenomena of this object by such a model.

The spectrum shown in Fig. 1 was taken on February 11, 1993, just at the phase of light minimum of the ephemeris of Doroshenko et al. (1993), which is the phase of apastron passage in their model. The spectrum shown in Fig. 5 was taken at a phase near light minimum. These spectra show deep and wide absorption components of $\mathrm{H} \mathrm{I}$ lines, which means that there was a strong mass outflow at these phases. Therefore, a large mass accretion to drive the outflow should continue even near the phase of apastron passage. Such a binary system, however, cannot hold an elliptical orbit during many orbital periods, because of the interaction between the two components. Panferov et al. (1997) supported the elliptical binary model and emphasized that long-period binary systems have, as a general tendency, elliptical orbits, but this is not the case for interactive binary systems. The binary system of this object may more likely have a circular orbit of which the period is not yet known.

The variation of the outflow velocity in the stable stage seems to be well fitted by a simple cosine curve (Fig. 6), and the phase of the minimum outflow velocity coincides with that of minimum luminosity. These results could be explained with a model of gas outflow from a precessing accretion disk. When the inclination of the disk to the line of sight is maximum, we will have minimum luminosity and minimum outflow velocity. On the other hand, when the accretion disk is faceon, the luminosity and the outflow velocity will become maxima. These phenomena are consistent with the observed results. Mikolajewski et al. (1997) reported a weak brightening of this object in $U B V$ bands in 1995, which may support our model, because the accretion disk was nearly face-on in that season. Unfortunately, such a brightening cannot be seen in our light curve (Fig. 6). This may be due to large observational errors, because our data include many visual estimates of different amateur astronomers.

Doroshenko et al. (1993) estimated the amplitude of the long-term light variation as 0.72 mag in the photographic band. If this variation was due to the precession of the disk, the maximum inclination of the disk to the line of sight should be about 59 degrees (see e.g. Webbink et al. 1987). Therefore, the angle of presession may be about 30 degrees. The open angle of the outflow should be larger than 59 degrees, because the outflow has been observed as absorption in all phases. However, the amplitude of the light variation being purely due to the precession of the disk seems to be still rather uncertain, because data on the outbursts are also included in the work of Doroshenko et al. (1993). If the amplitude of the light variation is $0.4 \mathrm{mag}$, the angle of precession may be about 23 degrees. A schematic model is shown in Fig. 7.

This model is reminiscent of another object, SS 433, where high velocity $(0.26 c)$ jets are observed (see e.g. Margon \& Anderson 1989). The behaviour of the moving emission components of H I, He I, etc. of SS 433 is explained with a model of a collimated high velocity jets from an accretion disk precessing with a period of about 163 days around a neutron star (Margon \& Anderson 1989 and references therein). If we substitute the neutron star in the model of SS 433 by a white dwarf, we will have an outflow velocity of the order of $1000 \mathrm{~km} \mathrm{~s}^{-1}$,

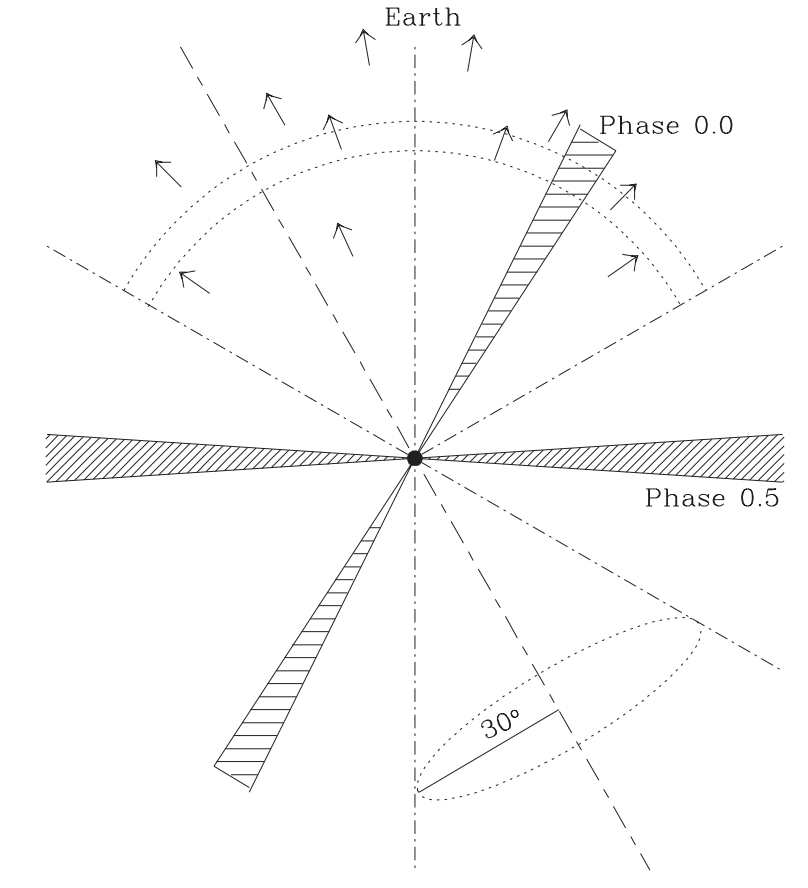

Fig. 7. A schematic model for the gas outflow from a precessing disk of MWC 560.

which is consistent with the observed results of MWC 560. Probably, there is a similar acceleration mechanism of gas outflow in MWC 560.

The angle of precession of the disk of SS 433 is estimated to be about 20 degrees (e.g. Margon \& Anderson 1989) from the variation of the radial velocities of the moving emission components. On the other hand, since the outflow of MWC 560 is observed as absorptions, we can see only the radial velocities of the outflowing matter in the line of sight. Therefore, the angle of precession cannot be estimated from the variation of the outflow velocity. Our results suggest that the outflow velocity decreases with increasing angular distance from the central axis of outflow.

If we represent the angular distribution of the outflow velocity as:

$v(\theta)=v_{0} \cdot \cos ^{\alpha} \theta$,

where $\theta$ is the angular distance from the central axis of outflow and $v_{0}$ is the outflow velocity at the central axis, we have $\alpha=0.48$ in the case of $\Delta m=0.72$, and $\alpha=0.87$ for $\Delta m=0.4$. These are preliminary results. Detailed photometric observations in $U$ and $B$ bands are awaited to estimate the amplitude of the light variation being purely due to the precession of the disk.

Similar wide-spread gas outflows are observed in some bipolar planetary nebulae, e.g. He2-104 and BI Cru (Corradi \& Schwarz 1993), but the outflow velocity of MWC 560 is more than ten times higher than those of these systems.

Figure 8 shows the radial velocities of the blue and red sides at half depth of the absorption components of $\mathrm{H} \alpha$ (open square and triangle) and $\mathrm{H} \beta$ (solid square and triangle). The dotted line in the upper panel is the best fit curve for $v_{\max }$, where the average velocity is arbitrarily shifted to fit the data. As seen in 


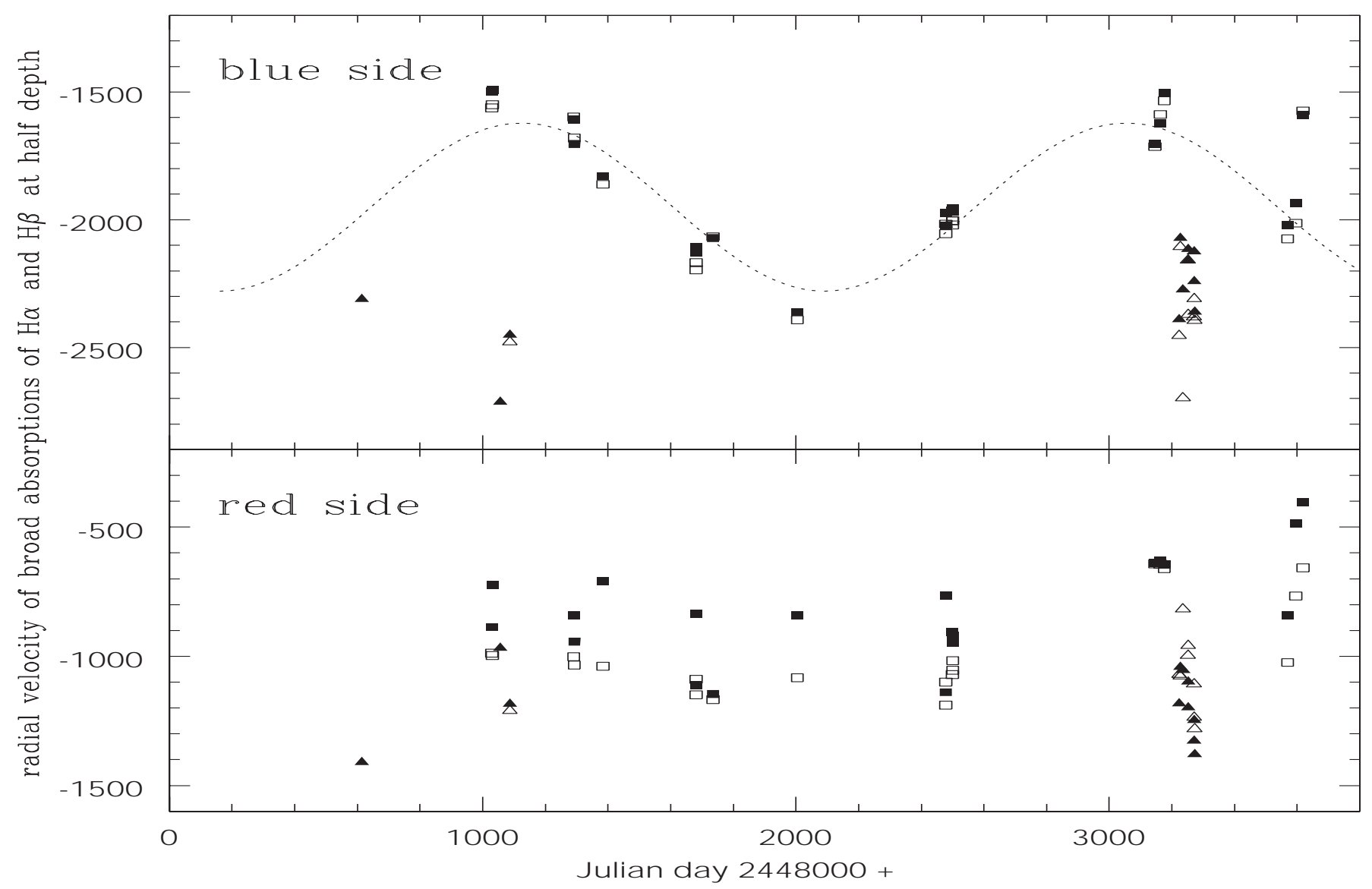

Fig. 8. Radial velocities in $\mathrm{km} \mathrm{s}^{-1}$ of the blue and red sides at half depth of the absorption components of $\mathrm{H} \alpha$ and $\mathrm{H} \beta$. The open square and triangle show the velocities of $\mathrm{H} \alpha$ in the stable and active stages respectively, while the solid symbols are those of $\mathrm{H} \beta$. The observational error is smaller than the symbols.

the figure, the variation of the radial velocity of the blue side is similar to that of $v_{\max }$, while large and rapid variations are seen in the velocity of the red side even in the stable stage. It seems that the low velocity part of the outflow is more unstable than the high velocity part. Schmid et al. (2001) also reported some different actions of the high velocity and low velocity parts of the outflow of this object.

\section{Active stage}

Here, we will point out only some phenomena in the active stage; a detailed analysis of the active stage will be made in a separate paper.

The profiles A and B (Figs. 1,2) were mainly observed in the stable stage. These profiles are rather similar to the classical P Cygni type absorptions. On the other hand, the profiles $\mathrm{C}$ and D (Figs. 3, 4) appeared only in the active stage. Some detailed investigations have been made of the profiles of the broad absorption components (e.g. Shore et al. 1994; Schmid et al. 2001), but the mechanism to present the flat continuum between the emission and absorption components is not yet well understood. At the present time we can only say that the outflow in the active stage may be very different from the normal gas outflow which presents classical P Cygni type absorp- tion. In the active stage, very high outflow velocities and their rapid variations were observed (Fig. 6 and see also Tomov et al. 1992 and Schmid et al. 2001). The acceleration of the outflowing matter may have been much stronger and more unstable in the active stage than in the stable stage.

Panferov et al. (1997) suggested that there are active and passive states in this object according to different conditions of the magnetic field of the hot component. They reported that the outflow velocity should be much higher and more rapidly variable in the active state than in the passive state. The properties of the outflow in their active state seem to be similar to those in our active stage. It is, however, not clear whether their active and passive states directly correspond to our active and stable stages, because the outflow velocity should be several hundred $\mathrm{km} \mathrm{s}^{-1}$ in their passive state (Panferov et al. 1997), while that in our stable stage is $-2100 \pm 330 \mathrm{~km} \mathrm{~s}^{-1}$.

During the outburst in 1990 this object was in the active stage, because very high outflow velocities and their rapid variations were observed, and the profiles of type C and D were seen in many spectra (Buckley 1992; Tomov et al. 1992). On the other hand, no significant light variation was seen in the active stages in 1993 and 1999 (Fig. 6). The active stage may not be necessarily accompanied by a brightening, at least in the $V$ band. The light curve given by Tomov et al. (1996) shows a 
weak brightening of this object at the beginning of 1993, but the active stage in 1993 started after the end of the brightening. The durations of the active stages were about several months.

The translation from the stable stage to the active stage occurs very rapidly. The profiles of $\mathrm{H} \delta$ obtained in the period December 1998-January 1999 by Schmid et al. (2001) show that a translation occurred between the days JD 2451178 and 179. During only one day, the profile of the absorption component of $\mathrm{H} \delta$ changed from type $\mathrm{A}$ to $\mathrm{D}$, and the outflow velocity increased approximately from $-1700 \mathrm{~km} \mathrm{~s}^{-1}$ to $-2100 \mathrm{~km} \mathrm{~s}^{-1}$. These velocities were processed on their Fig. 7 by the author.

The profile of the absorption component of $\mathrm{H} \gamma$ obtained by Mikolajewski et al. (1997) on September 19, 1996 is type D in our classification. Probably, there was another active stage in 1996, which was not monitored by our Observatory.

Temporal disappearances of the broad absorption components were observed in March-May 1990 during the outburst (Buckley 1992; Tomov et al. 1992). It may be unlikely that the disappearances were due to a failure of the pointing of the outflow to the line of sight, because the accretion disk should have been nearly face-on in those months. Probably, the outflow really ceased. Such a disappearance was not observed in the stable stage nor in the active stage during our monitoring. The cessation of the outflow may have been related to the outburst. Temporal cessations of outflow were observed also in SS 433 (e.g. Iijima 1993).

A very low outflow velocity was observed in February 1991 (Table 1, Fig. 6). Tomov et al. (1992) also reported similar low velocities in the same season. This phenomenon may have been related to the outburst, because such a low velocity was not observed later.

\section{Conclusion}

The behaviour of the broad absorption components of the $\mathrm{H}$ I lines in the stable stage is fairly different from that in the active stage. So, it is necessary to treat the data of the different stages separately in studies of this object. In the previous works, however, the difference between the data in the two stages was not well distinguished. Probably, this situation has made it difficult to understand the properties of this object.
The variation of the outflow velocity in the stable stage could be explained with a model of an outflow from an accretion disk precessing around a white dwarf with a period of 1930 days. This model is similar to that for the other high velocity outflowing object SS 433.

Since the outflow of MWC 560 is observed as absorption, we cannot estimate the angle of precession of the disk from the variation of the outflow velocity. However, when we will know the precise angle of precession from photometric data or by some other method, we will be able to find the angular distribution of the outflow velocity. MWC 560 may be the only object that presents us with such information. Further detailed experimental and theoretical works are required.

Acknowledgements. I am grateful to Prof. R. Barbon for the careful reading of the manuscript and useful suggestions.

\section{References}

Buckley, D. A. H. 1992, IAU Symp., 151, 421

Chentsov, E. L., Klochkova, V. G., \& Mal'kova, G. A. 1997, Bull. Spec. Astrophys. Obs., 43, 18

Corradi, R. L. M., \& Schwarz, H. E. 1993, A\&A, 268, 714

Doroshenko, V. T., Goranskij, V. P., \& Efimov, Y. S. 1993, IBVS, 3824

Iijima, T. 1993, ApJ, 410, 295

Iijima, T. 2000, ASP Conf. Ser., 204, 359

Margon, B., \& Anderson, S. F. 1989, ApJ, 347, 448

Michalitsianos, A. G., Maran, S. P., Oliversen, R.J., et al. 1991 ApJ, 371,761

Mikolajewski, M., Tomov, T., Georgiev, L., \& Jones, A. 1997, IBVS, 4519

Panferov, A. A., Fabrika, S. N., \& Tomov, T. 1997, Bull. Spec. Astrophys. Obs., 43, 37

Schmid, H. M., Kaufer, A., Camenzing, M., et al. 2001, A\&A, 377, 206

Shore, S. N., Aufdenberg, J. P., \& Michalitsianos, A. G. 1994, AJ, 108, 671

Szkody, P., \& Mateo, M. 1990, IAU Circ., 4987

Tomov, T., \& Kolev, D. 1997, A\&AS, 122, 43

Tomov, T., Kolev, D., Ivanov, M., et al. 1996, A\&AS, 116, 1

Tomov, T., Zamanov, R., Kolev, D., et al. 1992, MNRAS, 258, 23

Webbink, R. F., Livio, M., Truran, J. W., \& Orio, M. 1987, ApJ, 314, 653 